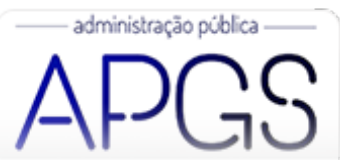

Administração Pública e Gestão Social ISSN: 2175-5787

apgs@ufv.br

Universidade Federal de Viçosa

Brasil

\title{
Qualidade Burocrática e Performance Estatal: Desvendando a caixa preta do município
}

Luis Rabelo Cardoso, André; Marenco, André

Qualidade Burocrática e Performance Estatal: Desvendando a caixa preta do município

Administração Pública e Gestão Social, vol. 11, núm. 4, 2019

Universidade Federal de Viçosa, Brasil

Disponível em: http://www.redalyc.org/articulo.oa?id=351560525001

Esta obra está bajo una Licencia Creative Commons Atribución-NoComercial-SinDerivar 3.0 Internacional 


\title{
Qualidade Burocrática e Performance Estatal: Desvendando a caixa preta do município
}

\author{
Bureaucratic Quality and State Performance: Unpacking the black box of the municipality \\ Calidad burocrática y desempeño estatal: Desentrañando la caja negra del municipio
}

André Luis Rabelo Cardoso

Universidade Federal do Rio Grande do Sul, Brasil

Redalyc: http://www.redalyc.org/articulo.oa?

Andreluis.mg@hotmail.com

André Marenco

Universidade Federal do Rio Grande do Sul, Brasil

amarenco@terra.com.br

Recepção: 21 Janeiro 2019

Aprovação: 13 Agosto 2019

Publicado: 01 Outubro 2019

\section{Resumo:}

A qualidade de uma burocracia local afeta os indicadores de desigualdade econômica em um município? Ela é capaz de melhorar os índices de desenvolvimento humano dessa população? Este trabalho é um esforço no sentido de compreender a estrutura burocrática do município, o ente governamental brasileiro responsável pelo processo de implementação da maioria das políticas públicas estatais. Com isso, pretende-se trazer à tona alguns traços característicos do perfil de uma burocracia municipal efetiva. Para tanto, após realizar uma redução de fatores, por meio da Análise Fatorial com dados no Munic 2005/10, foram feitas Correlações e Regressões Logísticas para testar: (1) Se a quantidade de servidores por habitante consegue explicar os índices obtidos em Gini e IDH-M, e (2) se a capacitação desses servidores é suficiente para explicar esses resultados, que evidenciam a importância da qualidade da burocracia local na contribuição para uma menor desigualdade econômica e melhor desenvolvimento humano.

Palavras-chave: Políticas Públicas, Burocracia, Implementação de Políticas Públicas, Capacidade Estatal, Administração Municipal.

\section{Abstract:}

Does the quality of a local bureaucracy affect the indicators of economic inequality in a city? Is it capable of improving the human development indexes of this population? This work is an effort to understand the bureaucratic structure of the Brazilian governmental entity that is the main responsible for the implementation process of most Brazilian state public policies: The municipality. With that, to bring to light some characteristic features of the profile of an effective municipal bureaucracy. To do so, after performing a factor reduction using Factorial Analysis with data in Munic 2005/10, Correlations and Logistic Regressions were made to test (1) if the number of servers per inhabitant can explain the indexes obtained in Gini and HDI-M coefficients and (2) whether the training of these servers is enough to explain these results, that show the importance of the quality of local bureaucracy in contributing to lower economic inequality and better human development.

KEYWORDS: Public policy, Bureaucracy, Implementation of Public Policies, State Capacity, Municipal Administration.

\section{Resumen:}

¿La calidad de una burocracia local afecta los indicadores de desigualdad económica en un municipio y puede mejorar las tasas de desarrollo humano en esta población? Este documento es un esfuerzo por comprender la estructura burocrática del municipio, la entidad gubernamental brasileña responsable por el proceso de implementación de la mayoría de las políticas públicas estatales. Con esto, se pretende sacar a la luz los rasgos característicos del perfil de una burocracia municipal efectiva. Por lo tanto, después de realizar una reducción de factores, a través del Análisis Factorial con datos en Munic 2005/10, se realizaron correlaciones y regresiones logísticas para probar (1) si el número de servidores por habitante puede explicar los índices obtenidos en coeficientes Gini y HDI-M, y (2) si la capacidad de estos servidores es suficiente para justificar estos resultados, que destacan la importancia de la calidad de la burocracia local para contribuir a reducir la desigualdad económica y mejorar el desarrollo humano. 
Palabras clave: Políticas públicas, Burocracia, Implementación de Políticas Públicas, Capacidad del estado, Administración municipal.

\section{INTRODUÇÃo}

Desde o surgimento dos primeiros agrupamentos humanos, o tema qualidade de governo tem sido objeto de busca e anseio social. $\mathrm{Na}$ academia, virou alvo da ciência política, da sociologia, da economia, além da administração; na prática, desejo de políticos, burocratas e também do cidadão comum, que deseja melhor qualidade de vida para si e sua família.

Nessa contínua busca por encontrar razões para explicação do subdesenvolvimento e, com isso, soluções para os entraves ao desenvolvimento, pesquisadores e teóricos recorreram aos mais diversos prismas analíticos. Inicialmente julgaram que as principais razões do atraso eram endógenas aos Estados, como a baixa escolaridade da população, elites conservadoras, estrutura agrária, etc. (Souza, 2017). Em seguida, argumentaram que eram as tecnologias, infraestrutura e capital de uma nação os maiores condicionantes de seu desenvolvimento econômico (Banco Interamericano de Desenvolvimento - BID, 2007).

Nota-se que, implicitamente, acreditava-se que a capacidade institucional viria a reboque dos recursos (BID, 2007). Dessa forma, durante muito tempo, a capacidade institucional foi percebida como um "resíduo não explicado" (Hirschman, 1967) e as instituições, encaradas como órgãos encarregados, simplesmente, de materializar as políticas e projetos. Nessa perspectiva, as soluções propaladas vinham ao encontro da lógica de que, para superar o atraso, era necessário copiar práticas, instituições e modelos adotados por países considerados desenvolvidos.

Entretanto, no final da década de 1970, após as crises do petróleo, o colapso do sistema financeiro internacional e os efeitos associados à III Revolução Industrial e à globalização, juntamente com a culminância dos paradigmas teóricos da escolha racional, da escolha pública e do agente/principal, propiciaram um contexto fecundo para o retorno de correntes defensoras do Estado mínimo e à adoção, pelo Estado, de práticas de mercado (Hood, 1991; Walker, Brewer, Boyne, Avellaneda, 2011). Intensificando ainda mais o debate sobre o funcionamento das instituições e sua influência nos resultados do desenvolvimento.

Todavia, já no final da década de 1980, avanços conceituais e analíticos de pesquisas sociais deram um novo rumo à discussão, trazendo à tona uma corrente denominada neo-institucionalismo, a qual passou a enfatizar, com mais veemência, o papel central das instituições, para explicar e influenciar os comportamentos sociais, econômicos e políticos (BID, 2007; Acemoglu \& Robinson, 2008; Evans \& Rauch, 1999; Hall \& Jones, 1999; North, 1990; Olson, 1996; Hall \& Taylor, 2003).

Com essa nova perspectiva, incorporava-se o entendimento de que os atributos do Estado faziam a diferença na implementação de políticas públicas, sendo requisito necessário ao seu sucesso, embora não suficiente. Sendo assim, a existência desses atributos, por si só, não garantiria um bom governo, mas seria condição à criação de mecanismos capazes de viabilizar o poder do Estado e fazê-lo materializar seus produtos e serviços (Souza, 2017). Entre esses atributos estaria a construção de burocracias profissionais. Tais contribuições constituíram o que ficou conhecido como Capacidade Estatal.

Seguindo essa linha de análise, este trabalho busca compreender um pouco mais sobre a estrutura burocrática do município brasileiro e, com isso, evidenciar alguns traços característicos do perfil de uma burocracia municipal efetiva. Assim, o tema de estudo é a burocracia, e a chave analítica é a construção de capacidades burocráticas para formulação e implementação de políticas públicas, associando-a a uma das vertentes do conceito de capacidade do Estado: A qualificação profissional da burocracia.

Cabe ressaltar que não se atreve aqui a buscar e propor modelos estruturais de burocracia bem-sucedidas sem considerar os arranjos institucionais locais, os quais são aqueles que, efetivamente, materializam as políticas públicas e que são resultados em graus variados de competências técnicas e políticas (Gomide \& 
Pires, 2014). Procura-se, de outra forma, dar enfoque específico a certas características institucionais, mesmo que, por vezes, não sejam estas completamente responsáveis por alguns de seus resultados, já que os resultados obtidos pelo Estado quase nunca são consequências de uma única variável.

Ainda há de se lembrar que, embora este seja um tema pouco estudado no Brasil, e menos ainda quando se trata de burocracias municipais, outras instituições e diversos autores reconhecidos já se enveredaram por desafios semelhantes, como, por exemplo, Pires (2009), Gomes e Avellaneda (2017), Grindle e Thomas (1989), Suzuki e Avellaneda (2018), Evans e Rauch (1999), Bo Rothstein (2011), Bardach (1977), Celina Souza (2015), Batista (2015).

Entretanto, antes de tentar compreender o perfil da estrutura burocrática de um município que consegue proporcionar qualidade de vida a seus cidadãos, é necessário definir os conceitos que subjazem a uma boa governança. Primeiramente, propôs-se um aprofundamento teórico sobre o tema; em seguida, fez-se um mapeamento de pesquisas que abordam o tema burocracia, as quais foram utilizadas como referencial teórico do trabalho. A partir daí, realizou-se a pesquisa, a fim de extrair as características das burocracias municipais de melhores desempenhos e, por fim, as considerações finais.

\section{Uma Revisão de Conceitos}

A literatura tem ressaltado certa confusão conceitual existente em relação à definição do que seria "qualidade de governo", "governança" e "capacidade estatal" (Fukuyama, 2013; Thomas, 2009; Andrews, 2008).

Por governo entende-se que é a instância máxima de administração executiva de um Estado/nação ou "o corpo decisório que exerce o poder de regrar uma sociedade política” (Rua, 1998, p. 70). O governo é um organismo que pode executar suas funções de melhor ou pior forma, e sua qualidade é determinada pelo impacto desse exercício de poder sobre a qualidade de vida dos seus cidadãos.

Mas o que promove o bom governo? Simplificadamente, diz-se que a qualidade de governança. Fukuyama (2013) define governança como a capacidade de o governo executar e fazer cumprir as regras para fornecimento de bens e serviços. Em razão disso, a governança tem relação com a execução ou, como frequentemente vem ocorrendo, tem recaído dentro do domínio da administração pública, em oposição à política.

Por outro lado, ao buscar seu significado em um dicionário, percebe-se que a palavra "governança" originase da palavra "governo" e implica alguma idéia de condução e controle. Dessa forma, é sensato pensar que governança está relacionada à capacidade de conduzir e controlar um sistema político.

Nesse sentido, o Banco Mundial apresentou uma definição de governança amplamente utilizada, como sendo "um conjunto de tradições e instituições através das quais a autoridade em um país é exercida para o bem comum" (Kaufmann, Kraay, Mastruzzi, 2004, p. 3). Bo Rothstein (2011, p. 8) complementa esse conceito, ao afirmar que ele acaba incluindo ""(1) o processo pelo qual os governos são selecionados, monitorados e substituídos, (2) a capacidade de o governo efetivamente formular e implementar políticas públicas sólidas, e (3) o respeito dos cidadãos para com as instituições que governam as interaçóes econômicas e sociais entre eles".

O que se entende por capacidade estatal? Diversas definições podem ser encontradas, ao se explorar esse conceito, por vezes incorporando variáveis políticas, institucionais, administrativas, coercitivas, legais, fiscais, territoriais, técnicas (Cingolani, 2013; Marenco, Strohschoen, Joner, 2016).

Marenco et al., (2016, p. 7), por exemplo, ressaltam o quanto a literatura sobre capacidade estatal tem sido fértil em empregar esse modelo para análises de problemas, tais quais, "controle da violência (Huntington, 1968), crescimento econômico (Evans, 1995; Evans \& Rauch, 1999; Fiani, 2014; Gomide, Silva \& Pires, 2014), prosperidade (Acemoglu \& Robinson, 2012), condições favoráveis para democracias (Tilly, 2007) ou qualidade de democracias (Rothstein, 2011)”. 
Isso demonstra que essas definições variam conforme a filiação teórica dos autores, seus propósitos analíticos e as respectivas perspectivas normativas sobre o papel do Estado (Cingolani, 2013; Pires \& Gomide, 2016). Marenco et al. (2016) ainda afirmam que essa amplitude analítica conferida ao conceito de “capacidades estatais” terminou por associá-lo a várias dimensões específicas, evidenciando claramente:

[...] uma sobreposição dos meios necessários para a provisão de capacidade estatal (coerção, leis, burocracia administrativa), com efeitos produzidos por sua disponibilidade, como ampliação da arrecadação fiscal, potencial para regulamentação do investimento, oferta de serviços públicos, legitimidade política (Marenco et al., 2016. p.7).

Já em outra perspectiva, Pires e Gomide (2016) e Jessop (2001) identificam duas gerações de análises sobre o assunto. A primeira, guiada pela compreensão dos processos históricos de formação dos Estados, traduzse pela necessidade de este criar e administrar um conjunto de estruturas relativas à proteção da soberania, coerção de condutas, administração de tributos e instituição de uma justiça. Fazem parte dessa geração os conceitos de capacidades estatais de autores como Tilly (1975), Skocpol (1979) e Levi (1988). Estes, por sua vez, possuem como característica comum: A tentativa de explicar, por meio do conceito, situações nas quais os Estados emergem, gerenciam conflitos internos e externos, e transformam as suas sociedades e economias (Pires \& Gomide, 2016).

A segunda geração de trabalhos procura discutir sobre as capacidades que o Estado possui, ou não, de atingir determinados objetivos, por intermédio de políticas públicas. Isto é, as capacidades de identificação de problemas, formulação de soluções, execução de ações e entrega dos resultados. Alinham-se a esta corrente trabalhos de autores como Skocpol (1985), Skocpol e Finegold (1982), Mann (1993) e Evans (1995).

Skocpol (1985), por exemplo, define capacidade estatal como a habilidade de o Estado implementar seus objetivos particularmente diante da oposição de grupos sociais poderosos, inserindo certa autonomia à burocracia para realização dos objetivos. Para Skocpol e Finegold (1982), capacidade do Estado significa que os governos têm ou podem mobilizar conhecimento, instituições e organizações capazes de implementar políticas. Mann (1984) a define como a capacidade de o Estado penetrar na sociedade e implementar, logisticamente, decisões políticas. Já Evans (1992) a conceitua como um conjunto de instrumentos e instituições de que dispõe o Estado para estabelecer objetivos, transformá-los em políticas e implementá-las.

Observe que, de forma ampla, a literatura sobre a capacidade estatal tem a característica de manifestar uma preocupação com as habilidades e competências do Estado, em estabelecer seus objetivos e implementálos, o que se aproxima da definição do substantivo "capacidade”, no Dicionário Aurélio, que está associada à "qualidade, habilidade ou aptidão que uma pessoa ou coisa tem de possuir para um determinado fim". Assim, capacidades estatais seriam as habilidades ou faculdades que o Estado precisa possuir para promover políticas públicas de qualidade, ou, como Peter Evans (1992) resumiu, a capacidade de promoção da ação do Estado.

Note que, de forma extremamente simplificada, a relação lógica entre os conceitos se assemelha ao exposto na Figura 01:

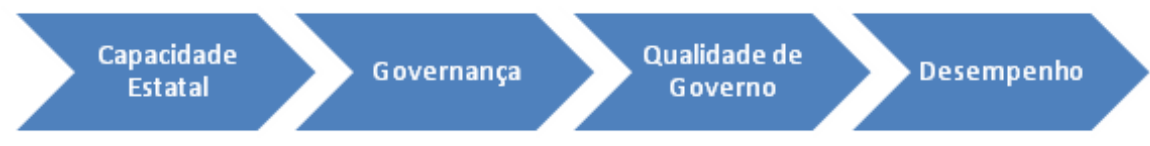

Figura 01: Simplificação lógica de relação entre os conceitos. Fonte: Elaborado pelos autores. 
Assim, pelos conceitos expostos, capacidade estatal é uma condição para uma boa governança, embora não suficiente para tal, mas parte fundamental dela (Ottervik, 2013). Isso sugere que a simples vontade de agentes ou atores políticos pode não ser suficiente para materialização dos objetivos pré-definidos. Da mesma forma, são os atributos dos Estados, que precisariam ser complementados por mecanismos que viabilizassem sua efetivação (Souza, 2017).

Por este trabalho, entende-se que a existência de capacidades estatais é requisito fundamental para o Estado alcançar seus objetivos. Ou, como Ottervik (2013) explica:

Os Estados de alta capacidade são capazes de fornecer bens públicos, como segurança, saúde e as infra-estruturas sociais e físicas que promovem o desenvolvimento humano (Rotberg, 2003). Os Estados de baixa capacidade são limitados em sua capacidade de fornecer esses bens, levando à baixa confiança social (Rothstein \& Stolle, 2008) e baixos níveis de desenvolvimento (Rotberg, 2004; Skocpol, 1979). Assim, para que a democracia seja consolidada com sucesso ao longo do tempo, é fundamental um Estado de alta capacidade (Wang, 2003; Fukuyama, 2005; Carothers, 2002) (Ottervik, 2013, p. 3 , tradução própria).

Nesta esteira de raciocínio, em que o Estado é posto em ação, ganha destaque a atuação da burocracia que, mesmo não sendo exclusiva na formulação e implementação de políticas públicas, via de regra, é a principal executora da vontade do Estado na provisão de bens e serviços. Portanto, capacidade estatal incorpora uma dimensão burocrática. A sua qualidade e profissionalização são as capacidades mais destacadas na literatura para predizer o que acontecerá com uma política pública (Souza, 2015).

\section{Um Mapeamento sobre Burocracia}

Se o poder de um Estado é proporcional a sua capacidade de traduzir suas idéias em ações (Kettl, 2006) e, se a atuação do Estado se concretiza, via de regra, pela atuação de sua burocracia (Oliveira \& Lotta, 2017), o poder de um Estado torna-se um poder burocrático, seja pela burocracia militar, seja pela burocracia de uma agência (Kettl, 2006), ou, de forma mais enfática, como Goodsell (2004) afirma, em interpretação extensiva, um governo sem uma burocracia poderosa passa a não ser um governo.

Se as burocracias possuem tamanho poder, dado que são elas os instrumentos das políticas do Estado, e se os burocratas também possuem poder semelhante, já que são eles quem decidem, no dia a dia, o que a burocracia irá realizar (Kettl, 2006), três perguntas acabam emergindo: Quem são esses burocratas? Quais são essas burocracias? E o que essas burocracias fazem?

Inicialmente, ressalta-se que os estudos tradicionais sobre o tema burocracia disseminam a ideia de uma burocracia homogênea, erigida sobre a concepção weberiana clássica, em que se valoriza aspectos como impessoalidade, hierarquia, seleção por mérito, cumprimento de regras e previsibilidade, e que tais atributos seriam indispensáveis para que o Estado cumprisse seu papel no desenvolvimento econômico e social (Bendix, 1964; Di Maggio \& Powell, 1983; Wilson, 1989; Carpenter, 2001; Peters, 2001; Kohli, 2004; Evans, 1995; Mommsen, 1989; Wade, 1990). Variações neste padrão de racionalidade burocrática foram analisados por Silberman (1993) incorporando incerteza como elemento para explicar discrepâncias observadas na administração pública contemporânea. Paralelamente, Evans (1995) e Evans \& Rauch (1999), revelaram associação entre burocracias profissionais e desenvolvimento econômico, Kohli (2007), BID (2010), Rothstein (2011), Holmberg \& Rothstein (2012), Acemoglu et al. (2015), Nistotskaya \& Cingolani (2016) e Meckling \& Nahm (2018), apresentaram elementos para evidenciar a relação entre racionalidade burocrática e qualidade na governança e políticas públicas.

Avanços na literatura têm trazido estudos, no sentido de que os burocratas, ainda que condicionados por contextos institucionais diversos, possuem alguma margem de ação para a realização de suas atividades cotidianas. Essas abordagens vão destacar o caráter heterogêneo da burocracia e sua relação com atores externos, marcada por negociações, reinterpretações de valores, acomodação de interesses e redefinição dos 
objetivos de uma política (Faria, 2003; Marques, 2003; Lotta; 2010; Pires, 2009; Lipsky, 1980; Wilson, 1968; Pressman \& Wildavsky, 1984).

Gabriela Lotta (2017)[1] faz um apanhado sobre essas pesquisas e as categoriza em cinco grupos distintos, quais sejam: (1) Compreender a atuação da burocracia na implementação em contexto de governança, (2) entender efeitos de elementos gerenciais na atuação da burocracia, (3) entender sobre o engajamento da burocracia em forma empreendedora ou ativista, (4) incorporar a dimensão das desigualdades na atuação da burocracia, e (5) compreender quais fatores influenciam a atuação da burocracia e de que forma se dá essa influência.

A primeira abordagem, numa tentativa de compreender a atuação da burocracia na implementação em contexto de governança, destaca temas como: (a) Pesquisas em estruturas de governança multinível (Hill \& Hupe, 2014; Klijn \& Koppenjan, 2000), (b) pesquisas que buscam compreender as práticas e papéis exercidos por burocratas de médio escalão (Abers, 2015; Oliveira \& Lotta, 2015; Pires, 2015; Cavalcante \& Lotta, 2015; Lotta, 2010; Oliveira \& Abrúcio, 2011; Lotta, Pires, Oliveira, 2014; Oliveira \& Lotta, 2017), (c) pesquisas sobre ocupação de cargos comissionados (Lopez \& Praça, 2015; De Bonis \& Pacheco, 2010), (d) pesquisas sobre intersetorialidade das políticas públicas (Bichir, 2015; Veiga \& Bronzo, 2014), (e) pesquisas que analisam a atuação da burocracia em interação com atores não estatais (Baird, 2016; Marques, 2013), (f) além de trabalhos tradicionais como Gomide e Pires (2014) e Gomide e Boschi (2016).

O segundo tipo de abordagem em pesquisas sobre burocracia busca entender efeitos de elementos gerenciais na atuação da burocracia. Destacam-se pesquisas de Perdicaris (2012), Prado (2012), Marin (2016), Assis (2012), Falcão-Martins (2006).

A terceira abordagem se ocupa do engajamento da burocracia de forma empreendedora ou ativista institucional (entendida como aquela integrante de movimentos sociais que, por ocuparem cargos no governo, buscam atingir os objetivos do movimento, utilizando-se dos canais oficiais). Nessa linha, destacamse Abers (2015), Abers, Serafim e Tatagiba (2014), Ferreira (2016).

A quarta abordagem tenta incorporar a dimensão da não uniformidade da atuação da burocracia. Neste caso, podem-se ressaltar trabalhos como os de Pires (2016) e Dubois (1999).

Por fim, a última categoria de pesquisas em burocracia, considerada por Lotta (2017), busca compreender de que maneira fatores podem influenciar a atuação da burocracia. Destacando autores como Pires (2009), Avellaneda e Gomes (2017), Grindle e Thomas (1989), Evans e Rauch (1999), Bo Rothstein (2011), Bardach (1977), Batista (2015) e Celina Souza (2015). Naturalmente, por se tratar da linha de pesquisa que orientará este trabalho, dar-se-á maior centralidade a ela.

Pires (2009) argumenta que as variações nos resultados de uma mesma política pública podem ser explicadas por diferentes práticas e estilos de execução adotados pelos inspetores do trabalho. Já os estudos de Avellaneda e Gomes (2017) argumentam que a capacidade gerencial do prefeito, avaliados pelo nível educacional e experiência, falham em explicar a variância da arrecadação de Imposto sobre a Propriedade Predial e Territorial Urbana (IPTU). Entretanto, fatores políticos (suporte legislativo e ciclo eleitoral) são mais bem correlacionados.

Grindle e Thomas (1989) trazem à tona como as barganhas entre os atores, em torno de recursos administrativos, poder e diferentes visões sobre uma política, podem afetar e remodelar os objetivos e os formatos organizacionais de políticas e programas.

Bo Rothstein (2011), por sua vez, desenvolveu um conjunto de medidas no intuito de apurar o "grau de imparcialidade das burocracias", que funcionou como uma proxy de qualidade de governança e, ainda, conseguiu correlacionar este índice com diversos indicadores de qualidade de vida.

Bardach (1977) desenvolveu uma tipologia de "jogos de implementação" que enfatiza os processos e os constrangimentos institucionais que criam oportunidades para desvio de recursos, atrasos, distorção e não realização de objetivos almejados. Para o autor, quanto mais simples for o processo de implementação, menores as oportunidades para desvio do planejado. 
Mariana Batista (2015), após estudar os efeitos da qualidade da burocracia local na implementação de políticas via recursos transferidos do Governo Federal, conclui que quanto maior a inadequação da burocracia, maior o número de falhas de implementação e de irregularidades na gestão de recursos federais; e quanto maior o número de funcionários por habitante e a proporção de funcionários com ensino superior, menor o número de falhas e de irregularidades na implementação desses recursos, confirmando os efeitos da qualidade da burocracia para explicar uma boa implementação de políticas.

Evans e Rauch (1999), a partir da aplicação de um questionário que capta um "grau de weberianidade" das nações, consegue correlacioná-lo aos níveis de crescimento da produção econômica das respectivas nações.

Celina Souza (2015), ao analisar a capacidade burocrática dos governos federais argentino e brasileiro, utilizando-se de um Índice de Qualidade Burocrática (IQB), o qual foi construído com base nas dimensões de profissionalização, qualificação, regras para promoção e forma de recrutamento da burocracia, conclui que o Brasil possui requisitos básicos weberianos (seleção via concursos e emprego com estabilidade). Entretanto, a Argentina manteve o recrutamento baseado nos laços pessoais e/ou partidários e sem estabilidade.

Assim, este trabalho foi baseado primordialmente no questionário desenvolvido e aplicado por Evans e Rauch (1999) e Evans (1992), bem como no índice de qualidade burocrática (IQB) desenvolvido por Celina Souza (2015), os quais podem ser igualmente utilizados para entender por que algumas políticas são mais bem-sucedidas que outras ou, neste caso, por que alguns municípios são mais ou menos capacitados tecnicamente para dar respostas a sua população local. Dessa forma, acredita-se contribuir ao criar possibilidades de se poder medir tecnicamente como a qualidade da burocracia possivelmente pode predizer alguns resultados de políticas públicas.

\section{Materiais e Métodos}

O argumento teórico utilizado neste trabalho é que os estudos sobre burocracias municipais, mais especificamente, sobre a atuação municipal como promotora de desenvolvimento e de welfare oferecem uma perspectiva promissora para reflexões sobre os desafios e soluções em termos de construção de capacidades estatais.

Nessa perspectiva, a qualidade das burocracias públicas municipais foram utilizadas como fator explicativo para o desempenho em implementação. Supondo-se, assim, que haveriam critérios ideais de composição da estrutura burocrática, que, quando observados, permitiriam melhorar o desempenho estatal.

De outro modo, trata-se de um estudo do tipo exploratório, com abordagem predominantemente quantitativa. Utilizando como fonte o banco de dados do grupo de pesquisa Instituições Políticas Comparadas da Universidade Federal do Rio Grande do Sul (IpoC/UFRGS) e a pesquisa do Perfil dos Municípios brasileiros (MUNIC), disponibilizadas pelo IBGE.

O objetivo final consiste em desvelar se algumas características clássicas das burocracias municipais repercutem em desempenho institucional, avaliados por meio de indicadores de contexto.

\subsection{Variáveis da Pesquisa}

A pesquisa propõe-se a estudar as variáveis abaixo no contexto municipal. 
Variáveis Explicativas

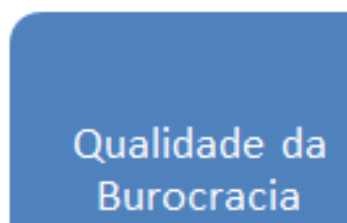

\section{Variável Dependente}

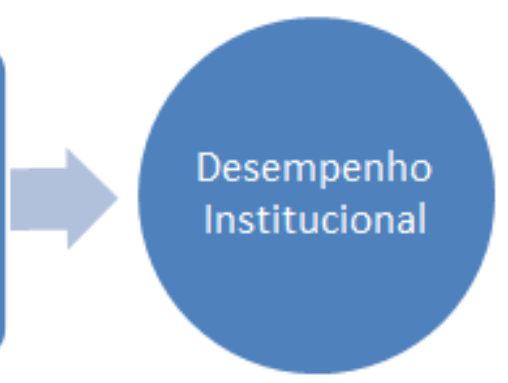

Figura 02: Variáveis exploradas.

Fonte: Elaborado pelos Autores.

\subsubsection{Qualidade da Burocracia}

A capacidade técnico-administrativa do município é operacionalizada por meio de quatro dimensões relacionadas aos critérios burocráticos weberianos típicos, os quais funcionaram como proxy para qualidade da burocracia.

Quadro 01: Dimensões da qualidade da burocracia.

\begin{tabular}{|c|c|c|c|}
\hline Dimensão & Indicador & Argumento & Sinal \\
\hline Quantitativo & $\begin{array}{l}\text { Quantitativo } \\
\text { de servidores } \\
\text { públicos } \\
\text { municipais por } \\
\text { habitante. }\end{array}$ & $\begin{array}{l}\text { Quanto maior a força de } \\
\text { trabalho, maior a } \\
\text { possibilidade de se obter } \\
\text { bons resultados. }\end{array}$ & + \\
\hline Recrutamento & $\begin{array}{l}\text { Percentual de } \\
\text { servidores } \\
\text { estatutários. }\end{array}$ & $\begin{array}{l}\text { Carreiras de longo prazo } \\
\text { e com previsibilidade; } \\
\text { Regras para contratar e } \\
\text { demitir que substituam } \\
\text { contratações e } \\
\text { demissões arbitrárias; } \\
\text { Contratação baseada no } \\
\text { mérito; Profissionais } \\
\text { mais livres de } \\
\text { ingerências politicas. }\end{array}$ & + \\
\hline Qualificação & $\begin{array}{l}\text { Capacitação } \\
\text { dos servidores } \\
\text { por meio da } \\
\text { escolaridade. }\end{array}$ & $\begin{array}{l}\text { Quanto maior o grau de } \\
\text { instrução, melhores os } \\
\text { resultados alcançados. }\end{array}$ & + \\
\hline Recrutamento & $\begin{array}{l}\text { Proporção de } \\
\text { servidores com } \\
\text { contratos } \\
\text { temporários }\end{array}$ & $\begin{array}{l}\text { Profissionais mais } \\
\text { Suscetiveis a influências } \\
\text { externas; Maior } \\
\text { possibilidade de } \\
\text { politização negativa da } \\
\text { adm. pública. }\end{array}$ & - \\
\hline
\end{tabular}

Fonte: Elaborado pelos autores. 


\subsubsection{Desempenho Municipal}

Utilizou-se duas variáveis funcionando como proxy de medição de desempenho municipal. A primeira delas foi o Índice de Desenvolvimento Humano Municipal (IDH-M) e a segunda, o índice Gini. O IDH-M, funcionou como uma proxy de eficácia governamental, e o Gini, como proxy do grau de concentração de renda, ou seja, da equidade resultante da ação pública municipal ${ }^{[2]}$.

A partir desses resultados ranqueou-se os municípios.

\subsection{A Escolha dos Casos e Métodos}

Não se pretendeu estudar todos os municípios brasileiros, dado o tamanho da variabilidade estrutural que estes possuem. Fato que dificultaria a operacionalização dos conceitos. Assim, a fim de facilitar a manipulação das variáveis e melhor isolar o efeito direto delas, foram utilizados os dados dos municípios categorizados pelo IBGE como 1, 2 e 3 (ou seja, até 20.000 habitantes). Além disso, optou-se por operacionalizar o trabalho a partir de dois processos distintos para seleção dos casos.

No primeiro processo, foram escolhidos para composição da amostra os 100 municípios que obtiveram os maiores valores no indicador IDH-M de 2010 e outros 100 que obtiveram os piores índices no mesmo indicador. Esta estratégia se deu em razão da necessidade de se condensar, na mesma amostra, os casos polares.

Já no segundo processo de seleção, seguindo a mesma lógica anterior, selecionaram-se os 100 municípios que obtiveram os maiores valores no indicador Gini de 2010 e os 100 que obtiveram as piores avaliações.

Assim, foram comparadas a estrutura burocrática dessas duas amostras de 200 municípios cada, as quais foram compostas, basicamente, por casos polares. Ressalta-se que este quantitativo selecionado não foi aleatório, mas pensado para atender aos requisitos do quantitativo mínimo por variável dependente por ferramenta de análise.

Nota-se que esses municípios selecionados possuem tamanhos populacionais similares, sendo muito pequenos, se comparado aos grandes centros urbanos. Mas, ainda assim, tendo resultados bastante diferentes no que tange ao desempenho estatal.

\subsection{Procedimentos para análise dos dados}

Para analisar estes dados foram utilizados três ferramentas distintas, a saber:

- Análise Fatorial: Representa um processo aleatório multivariado por meio da criação de novas variáveis, derivadas das variáveis originais e, geralmente, em menor número, que representa a comunalidades do processo. É uma ferramenta complexa, mas fundamental para facilitação do uso de outras ferramentas;

Correlação de Pearson: Se duas ou mais variáveis são associadas, diz-se que são correlacionadas. Isso significa que co-variam, ou seja, quando os valores em uma variável mudam, valores da outra variável também mudam. $\mathrm{O} \rho$ de Pearson mensura a magnitude e grau de relacionamento e a probabilidade de tal relacionamento ocorrer;

Regressão Logística Binária: É uma forma específica de regressão que é formulada para prever e explicar uma variável categórica binária (dois grupos), e não uma medida dependente métrica. Neste tipo de análise, as variáveis dependentes são dispostas em categorias dicotômicas (sim, não; sucesso, fracasso) e a resposta na Regressão Logística é expressa por meio de uma probabilidade de ocorrência (Hear, Anderson, Tatham, Black, 2005). Destaca-se ainda que a Regressão Logística não 
depende de suposições rígidas (como normalidade multivariada e igualdade de matrizes de variâncias e covariâncias), tornando-a ainda mais robusta quando esses pressupostos não podem ser atendidos.

Assim, de forma sucinta, inicialmente foi feita uma redução das dimensões das variáveis relativas à quantidade de servidores por habitante e a capacitação dos servidores municipais, utilizando-se da Análise Fatorial. Em seguida, foram rodadas as Correlações e Regressões Logísticas Binárias, utilizando-se dos fatores extraídos, numa tentativa de explicar o resultado municipal relativo ao IDH-M e Gini obtidos em 2010.

A partir desses resultados, desdobrou-se a análise para avaliar se o tipo de vínculo que estes servidores possuíam com a administração municipal também seria relevante para explicar tal resultado. Para tal, utilizouse dos dados do MUNIC/IBGE anteriores ao ano de 2010, ou seja, os dados relativos ao perfil dos servidores municipais disponibilizados e referentes aos anos de 2005, 2006, 2008 e 2009[3].

\section{Resultados e Discussão}

O universo dos municípios analisados são aqueles com população inferior a 20.000 habitantes. Sendo assim, englobam os municípios categorizados pelo IBGE como: (1) Com até 5.000 habitantes, (2) entre $5.001 \mathrm{e}$ 10.000 habitantes, e (3) entre 10.001 e 20.000 habitantes. Dentre estes, o menor deles é Serra da Saudade (MG), com 812 pessoas, e o maior, Rubiataba (GO), que possui 19.994 habitantes. O total de habitantes neste universo é de pouco mais de 32 milhões de pessoas.

De forma geral, ao analisar a Figura 3, nota-se que o quantitativo de municípios categorizados como “1", “2” e “3” são bastantes próximos uns dos outros (1235, 1215 e 1352, respectivamente), entretanto, o quantitativo populacional que eles concentram é bastante divergente (13\% categoria " 1 ", $26,9 \%$ classe "2" e 60,1\% classificação "3”, desse universo).

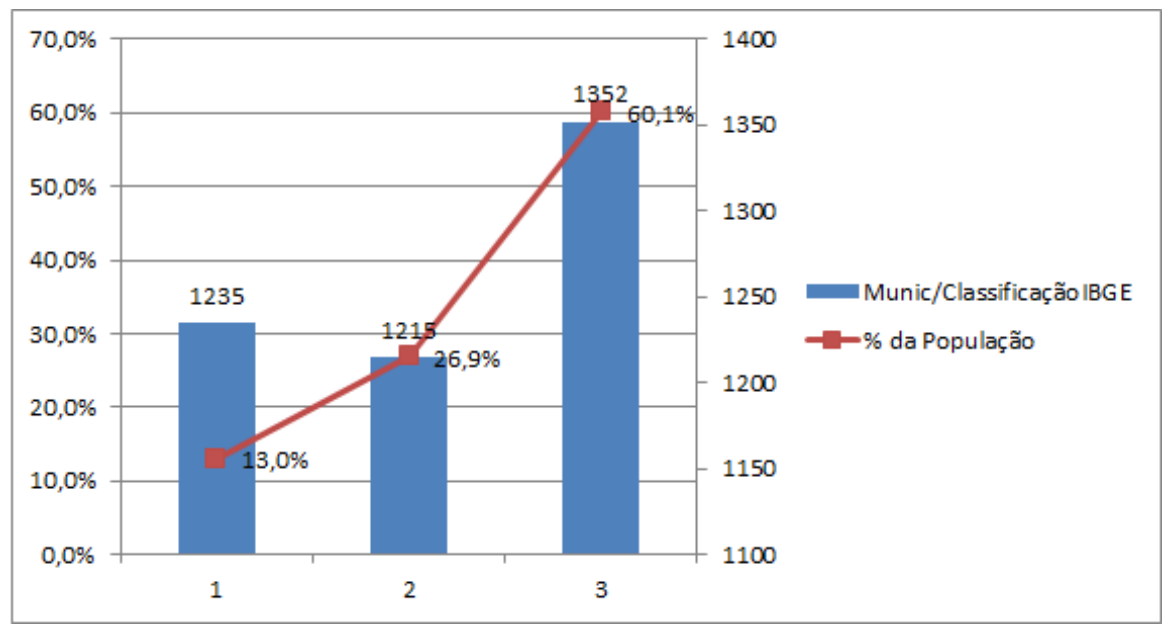

FIGURA 03: Município, Tamanho e População. Fonte: MUNIC/IBGE, iPOC-UFRGS.

$s$ amostras analisadas, conforme explicitado, foram compostas por 200 municípios cada, sendo os 100 maiores e menores valores em IDH-M. Procedeu-se da mesma maneira para os dados relativos ao Gini (lista de municípios anexa).

Inicialmente procedeu-se à Análise Fatorial pelo método dos componentes principais sobre as variáveis relativas ao: (1) Quantitativo total de servidor/quantidade de pessoas do município (2005, 2006, 2008 e 2009) e (2) média ponderada da capacitação total dos servidores/quantidade servidores da administração direta total (2005 e 2008).

Cabe explicitar, portanto, que este último coeficiente, denominado "média ponderada da capacitação total dos servidores dividido pela quantidade de servidores da administração direta total”, trata-se de um fator 
resultante da média aritmética ponderada dos quantitativos de servidores aplicados a pesos de acordo com a formação. Em seguida, dividiu-se esse número pelo quantitativo de servidor da administração direta do município, conforme fórmula a seguir:

$$
\frac{\frac{((\operatorname{Serv} . \operatorname{SemInst} * 1)+(\operatorname{Serv} . \text { Fund } * 2)+(\operatorname{Serv} . \text { Méd } * 3)+(\operatorname{Serv} .4)+(\operatorname{Serv} . \text { Pós } * 5))}{15}}{\text { Total.ServidoresAdministraçãoDireta }}
$$

Para rodar a Análise Fatorial, optou-se por extrair apenas uma variável como fator para cada grupo de dados. Isto porque tratam-se de variáveis de mesma natureza e que foram obtidas ano após ano dos mesmos municípios, alterando apenas os quantitativos, o que faz com que, naturalmente, elas tenham grande probabilidade de ter altos valores de comunalidades. Seguem alguns dos resultados mais relevantes na análise:

Quadro 02: Resultados Análise Fatorial.

\begin{tabular}{|c|c|c|c|}
\hline & Variável & Quantit.Serv./Total.Pop & $\begin{array}{l}\text { Média pond. } \\
\text { capacit. } \\
\text { servid/quantid. } \\
\text { Serv. }\end{array}$ \\
\hline \multirow[t]{3}{*}{ IDH-M } & KMO & 0,733 & 0,50 \\
\hline & Sig. & 0,000 & 0,000 \\
\hline & $\begin{array}{l}\text { \%Comunalidades } \\
\text { Extraídas }\end{array}$ & $82,44 \%$ & $75,64 \%$ \\
\hline \multirow[t]{3}{*}{ Gini } & $\mathrm{KMO}$ & 0,814 & 0,50 \\
\hline & Sig. & 0,000 & 0,000 \\
\hline & $\begin{array}{l}\text { \%Comunalidades } \\
\text { Extraídas }\end{array}$ & $88,21 \%$ & 66,72 \\
\hline
\end{tabular}

Fonte: Elaborado pelos Autores.

Quanto aos coeficientes do teste anti-imagem, estes se mostraram em todas as variáveis superiores a 0,5. Além disso, o requisito de se ter em uma amostra um mínimo de observações 5 vezes maior que o número de variáveis também foi atendido. Em seguida, utilizando-se das variáveis independentes extraídas e relativas a "porcentagem da quantidade de servidores total/população" e a "média capacitação desses servidores/total de servidores", procedeu-se a Análises de Correlação.

Pelo fato de as variáveis possuírem distribuição normal (Shapiro-Wilk dados Gini= p<0,001; e IDH-M= $\mathrm{p}<0,001)$, um dos requisitos básicos para a Análise de Correlação de Pearson, procedeu-se este teste.

A Correlação de Pearson mostrou que há uma correlação positiva e moderada entre a capacidade dos servidores e o desempenho no IDH-M $(p=0,58 ; \mathrm{p}<0,001)$. Já quantidade de servidores municipais por habitantes não se mostrou correlacionada ao referido desempenho.

Em relação aos dados relativos ao Gini, o mesmo resultado foi encontrado entre a "capacidade dos servidores" e o referido desempenho $(p=-0,30 ; p<0,001)$. A quantidade de servidores municipais por habitante também não se correlacionou com o desempenho.

Posteriormente, com essas mesmas variáveis extraídas pela Análise Fatorial, operacionalizou-se a Regressão Logística Binária. Para tanto, para aqueles municípios que possuíam valores mais altos de IDH-M e Gini foram atribuídos o valor "1", e para os que possuíam os valores baixos, o número " 0 ".

Ao verificar se a "quantidade" e "capacitação dos servidores" são bons previsores para o resultado do IDH$\mathrm{M}$, o modelo se mostrou significativo $\left[\mathrm{X}^{2}(8)=10,62 ; \mathrm{p}<0,005 ; \mathrm{R}^{2}\right.$ Negelkerke $\left.=0,39\right]$, sendo a capacitação dos servidores um bom previsor do resultado do IDH-M (OR = 5,73; IC 95\% = 3,31 - 9,93). 
De igual maneira, procedeu-se análise com os dados do Gini, e o modelo também se mostrou significativo $\left[X^{2}(8)=9,12 ; p<0,001 ; R^{2}\right.$ Negelkerke $\left.=0,135\right]$. Nesse caso a capacitação dos servidores foi um previsor do resultado do Gini $(\mathrm{OR}=0,46$; IC 95\% = 0,31 - 0,67).

Em ambos os testes os resultados indicam que quanto mais capacitada a força de trabalho municipal, maior a probabilidade de ela ter bons resultados no IDH-M e Gini. Entretanto, o mesmo não ocorreu com a variável "quantidade de servidores municipais por habitante", pois em ambas as análises elas não se mostraram significativamente relevantes na explicação, contrariando os achados de Batista (2015), ao encontrar em seus estudos menor número de falhas e irregularidades na implementação de recursos federais para aqueles municípios que possuíam maior número de funcionários por habitante e a proporção com ensino superior.

Diante dessa evidência, buscou-se desdobrar a variável "capacitação dos servidores municipais" de acordo com o vínculo que estes servidores possuíam com a administração pública municipal.

De igual maneira, procedeu-se à Análise Fatorial dos seguintes dados: (1) Média ponderada da capacitação dos servidores estatutários/quantidade de servidores ligados à administração direta (2005/08), (2) Média ponderada da capacitação dos servidores cujo trabalho é regido pela Consolidação das Leis do Trabalho (CLT)/quantidade de servidores ligados à administração direta (2005/08), (3) Média ponderada da capacitação dos servidores comissionados/quantidade de servidores ligados à administração direta (2005/08) e (4) Média ponderada da capacitação dos servidores sem vínculos/quantidade de servidores ligados à administração direta (2005/08). Seguem os principais resultados:

Quadro 03: Resultados Análise Fatorial.

\begin{tabular}{|c|c|c|c|c|c|}
\hline & Variável & $\begin{array}{l}\text { Capacidade } \\
\text { Estatutários/Serv. }\end{array}$ & $\begin{array}{l}\text { Capacidade } \\
\text { CLT / Serv. }\end{array}$ & $\begin{array}{l}\text { Capacidade } \\
\text { Comissionado/Serv. }\end{array}$ & $\begin{array}{l}\text { Capacidade } \\
\text { Sem } \\
\text { Vinculo/Serv. }\end{array}$ \\
\hline \multirow[t]{3}{*}{ IDH-M } & $\mathrm{KMO}$ & 0,5 & 0,5 & 0,5 & 0,5 \\
\hline & Sig. & 0,000 & 0,000 & 0,000 & 0,000 \\
\hline & $\begin{array}{l}\% \\
\text { Comunalidades } \\
\text { Extraidas }\end{array}$ & $85,08 \%$ & $82,8 \%$ & $80,2 \%$ & $62,43 \%$ \\
\hline \multirow[t]{3}{*}{ Gini } & $\mathrm{KMO}$ & 0,5 & 0,5 & 0,5 & 0,5 \\
\hline & Sig. & 0,000 & 0,000 & 0,000 & 0,000 \\
\hline & $\begin{array}{l}\text { \%Comunalidades } \\
\text { Extraídas }\end{array}$ & $83,24 \%$ & $88,99 \%$ & $76,10 \%$ & $77,61 \%$ \\
\hline
\end{tabular}

Fonte: Elaborado pelos autores.

Dando sequência, procedeu-se à Regressão Logística Binária com os fatores extraídos.

Ao verificar se a capacitação dos servidores municipais de acordo com o vínculo que estes possuíam com a administração pública (Estatutários, CLT, Comissionados ou Sem vínculos) eram bons previsores para o resultado do IDH-M, o modelo se mostrou significativo $\left[X^{2}(4)=28,69 ; \mathrm{p}<0,001 ; \mathrm{R}^{2}\right.$ Negelkerke $\left.=0,20\right]$.

Neste caso, tem-se os dados para a capacitação dos servidores "Estatutário" previsores do resultado do IDH-M $(\mathrm{OR}=0,16$; IC 95\% = 0,072 - 0,39), a capacitação dos vinculados pela "CLT" (OR = 0,42; IC $95 \%=0,22-0,82)$, a capacitação dos vinculados "Somente Comissionados" (OR = 1,47; IC 95\% = 1,03 2,10) e "Sem Vínculos" também como previsor do resultado (OR = 0,40; IC 95\% = 0,23 - 0,72).

Ressalta-se que a significância encontrada para dos servidores "Estatutários", se mostrou maior que os demais vínculos na explicação dos resultados obtidos pela administração pública municipal. 
Por outro lado, ao testar as variáveis relativas à capacitação dos servidores de acordo com o vínculo administrativo com o desempenho relativo ao Gini, o modelo não se mostrou significativo em nenhuma das classes.

Dessa forma, a equação que descreve a relação é:

$$
\begin{aligned}
& P(\text { IDH }-M) \\
& =\frac{e^{-1,78(\text { CapEst } / \text { serv })-0,85(\text { CapCLT } / \text { serv })-0,38(\text { CapComis } / \text { ser })-0,89(\text { CapSemvinc } / \text { serv })}}{1+e^{-1,78(\text { CapEst } / \text { serv })-0,85(\text { CapCLT } / \text { ser })-0,38(\text { CapComis } / \text { ser })-0,89(\text { CapSemvinc/serv })}}
\end{aligned}
$$

Em resumo, os testes evidenciam o quanto a capacitação dos servidores públicos municipais é importante para o retorno em forma de qualidade de vida do cidadão, e mais, o quão importante é o tipo de vínculo que esse servidor possui com a administração pública, principalmente pelo fato de o vínculo "estatutário" ter se mostrado mais significativo na explicação dos resultados. Sugere-se, assim, que a forma de seleção do servidor é naturalmente um diferencial e previsor dos resultados por ele alcançados, corroborando com os preceitos recomendados por Weber, desde o início do século, para a construção de uma burocracia eficaz e efetiva.

\section{Considerações Finais}

Este trabalho buscou compreender a estrutura burocrática da administração pública local. Neste sentido, a qualidade da burocracia por meio das dimensões "quantidade de servidores por habitante" e "capacitação dos servidores" foram operacionalizadas para tentar explicar os resultados obtidos por estes municípios no Gini e IDH-M.

Os resultados alcançados por meio das correlações e regressões logísticas evidenciam o quanto a capacitação dos servidores públicos municipais é importante para que a administração pública alcance bons resultados. Também como resultado destaca-se o papel dos servidores estatutários, dado que sua capacitação foi mais relevante na obtenção do indicador IDH-M que os demais vínculos administrativos. Entretanto, diferentemente do esperado, o quantitativo de servidores por habitante não se mostrou significativo em nenhuma das amostras.

Como maior limitação do trabalho, ressaltam-se as variáveis utilizadas como proxy de desempenho, as quais possuem a características de não serem produzidas, única e exclusivamente, como fruto da ação de um único agente, mas podem ser proporcionadas por diversos atores, tanto públicos como privados. Isso, naturalmente, abre a possibilidade para questionamentos decorrentes de a ação estatal não ter sido sua principal causadora, mas de outras ações não identificadas.

Obviamente, algumas questões não foram abordadas aqui, detalhadamente, como, por exemplo, o efeito da capacitação dos prefeitos ou mesmo dos seus secretários comissionados neste resultado. O que não deixa de ser uma grande oportunidade para outras pesquisas.

Por fim, acredita-se que este seja um passo inicial na busca por compreender o funcionamento e os resultados obtidos por uma burocracia pública municipal, mas que pode e deve ser alvo de outras análises nos estudos sobre políticas públicas no Brasil, principalmente diante dos retornos que estes resultados têm o potencial de oferecer ao cidadãos e gestores públicos.

\section{RefERÊNCIAS BibLIOgRÁficas}

Abers, Rebecca Neaera. (2015). Capítulo 5 - Ativismo na burocracia? O médio escalão do programa bolsa verde. In: Pedro Cavalcante e Gabriela Lotta. (Org.). Burocracia de Médio Escalão: perfil, trajetória e atuação. 1 ed. v. , p. 143-176. Brasília: ENAP. 
; Serafim, Lizandra; Tatagiba, Luciana. (2014). Repertórios de Interação Estado-Sociedade em um Estado Heterogêneo: a Experiência na Era Lula. Dados - Revista de Ciências Sociais. Rio de Janeiro, vol. 57, n. 2, p. $325-35$.

Acemoglu, Daron; Robinson, James. (2012). Why Nations Fail? The origins of power, prosperity and poverty. New York: Crown.

; Garcia-Jimeno, Camilo. (2015). State Capacity and Economic Development: A Network Approach. American Economic Review . Vol 105(8): 2364-2409.

Andrews, Matt. (2008). The Good Governance Agenda: Beyond Indicators without Theory. Oxford Development Studies. 36 (4): 379-407.

Assis, Luís Otávio Milagres de. (2012). Efeitos Organizacionais da Implantação de Metas e Remuneração Variável por Desempenho: o caso da segurança pública em Minas Gerais. Dissertação (Mestrado) - Escola de Administração de Empresas de São Paulo da Fundação Getúlio Vargas. São Paulo - SP. p.177.

Batista, Mariana. (2015). Burocracia local e qualidade da implementação de políticas descentralizadas: uma análise da gestão de recursos federais pelos municípios brasileiros. Revista do Serviço Público. Brasília; v. 3, n. 66, p. 345-370, jul.

Bendix, Reinhard. (1964). Nation-Building and Citizenship: Studies of four changing social order. John Wiley and Sons.

Bichir, Renata Mirandola. (2015). Capacidades Estatais Para a Implementação de Programas de Transferência de Renda: Os Casos de Brasil, Argentina e África do Sul. Texto Para Discussão-TD 2032. IPEA. Rio de Janeiro, jan.

BID (Banco Interamericano de Desenvolvimento). (2007). A política das políticas públicas: progresso social e econômico na América Latina. Relatório 2006 do BID e do David Rockfeller Center for Latin American Studies, Harvard University. Rio de Janeiro: Elsevier; Washington, DC: BID.

Carothers, Thomas. (2002). The End of the Transition Paradigm. Journal of Democracy. 13 (1): 5-21.

Carpenter, Daniel. (2001). The Forging of Bureaucratic Autonomy: Reputations, Networks, and Policy Innovation in Executive Agencies. 1862-1928. Princeton University Press.

Cavalcante, Pedro; Lotta, Gabriela. (2015) Burocracia de Médio Escalão: perfil, trajetória e atuação. Brasília: ENAP.

Cingolani, L. (2013). The state of state capacity: a review of concepts, evidence and measures. Maastricht: UNOMERIT.

Dancey, Christine P. Reidy, John. (2006). Estatística sem Matemática para Psicologia. Usando SPss para Windows. Porto Alegre: Artmed.

De Bonis, Daniel; Pacheco, Regina. (2010). Nem político, nem burocrata: o debate sobre o dirigente público, in: M.R. Loureiro; F. Abrucio; R. Pacheco (Orgs.) Burocracia e Política no Brasil. Desafios para o Estado democrático no século XXI. Rio de Janeiro, Fundação Getúlio Vargas, p. 329-62.

Di Maggio, Paul J.; Powell, Walter W. (1983). The Iron Cage Revisited: Institutional Isomorphism and Collective Rationality in Organizational Fields. American Sociological Review, Vol. 48, No. 2, pp. 147-160.

Dubois, Vincent. (1999). La vie au guichet. Relation administrative et traitement de la misère. Paris: Economica, coll. Études politiques.

(2010). The bureaucrat and the poor: encounters in French welfare offices. Burlington: Ashgate.

Evans, Peter B. (1992). The state as problem and solution: predation, embedded autonomy, and adjustment. In: Haggard, Stephan; Kaufman, Robert R. (Orgs.). The politics of economic adjustment. Princeton, NJ: Princeton University Press. p. 139-191.

(1995). Embedded Autonomy. Princeton: Princeton University Press.

Evans, Peter; Rauch, J. E. (1999). Bureaucracy and growth: A cross-national analysis of the effects of "Weberian" state structures on economic growth. American Sociological Review. 64(5):748-765.

Evans, Tony. (2011). Professionals, Managers and Discretion: Critiquing Street-Level Bureaucracy. The British Journal of Social Work, Volume 41, Issue 2, 1. p. 368-386. Março. 
Falcão-Martins, H. (2006). Em Busca da Integração entre Planejamento e Gestão: a Abordagem do Governo Matricial em Síntese. Saber, Revista da Escola de Governo do Estado do Pará, v. 01, p. 1-2.

Faria, Carlos Aurélio Pimenta de. (2003). Ideias, conhecimento e políticas públicas: um inventário sucinto das principais vertentes analíticas recentes. Revista Brasileira de Ciências Sociais, 18 (51): 21-30.

Ferreira, Daniela Damiati. (2016). Ativismo Institucional no Governo Federal: as Práticas dos Analistas Técnicos de Políticas Sociais. Dissertação Mestrado - Pós Graduação em Políticas Públicas da Universidade Federal do ABC. São Bernardo do Campo- SP. p. 153.

Fiani, Ronaldo. (2014). Arranjos institucionais e desenvolvimento: o papel da coordenação em estruturas híbridas. IN: Capacidades estatais e democracia : arranjos institucionais de políticas públicas. editores: Alexandre de Ávila Gomide, Roberto Rocha C. Pires. - Brasília : Ipea.

Fukuyama, Francis. (2005). Stateness First. Journal of Democracy. 16(1): 84-88.

. (2013). What is governance?. Governance. v. 26, n. 3, p. 347-368.

Gomes, Ricardo Corrêa; Avellaneda, Claudia Nancy. (2017). Mayoral Quality and Municipal Performance in Brazilian Local Governments. O\&S - Salvador, v. 24, n. 83, p. 555-579, Out./Dez.

Gomide, Alexandre de Ávila; Pires, Roberto Rocha C. (2014). Capacidades estatais e democracia: arranjos institucionais de políticas públicas/ Editores: Alexandre de Ávila Gomide, Roberto Rocha C. Pires. - Brasília: Ipea.

Gomide, Alexandre de Ávila; Boschi, Renato Raul. (2016). Capacidades estatais em países emergentes: o Brasil em perspectiva comparada / Editores: Alexandre de Ávila Gomide, Renato Raul Boschi. - Rio de Janeiro: Ipea.

Gomide, Alexandre; Silva, Fábio; Pires, Roberto. (2014). Capacidades estatais e políticas públicas: passado, presente e futuro da ação governamental para o desenvolvimento. IN: Brasil em desenvolvimento 2014: estado, planejamento e políticas públicas. editores: Leonardo Monteiro Monasterio, Marcelo Côrtes Neri, Sergei Suarez Dillon Soares. Brasília: Ipea.

Goodsell, C. T. (2004). The Case for Bureaucracy: A Public Administration Polemic. 4th ed ${ }^{\circ}$. Washington, DC: CQ Press.

Hear, Jr. J. F.; Anderson, R.E.; Tatham, R.L.; Black, W. C. (2005). Análise multivariada de dados. 5. ed., Porto Alegre: Bookman.

Hill, Michael; Hupe, Peter. (2014). Implementing Public Policy. Los Angeles: SAGE.

Hirschman, Albert O. (1967). Development Projects Observed. Washington, DC: Brookings Institution Press.

Holmberg, Soren; Rothstein, Bo. (2012). Good Government. The relevance of political Science. Northampton, Elgar.

Hood, Christopher. (1991). A public management for all seasons?. Public administration. 69 (1):3-19.

Huntington, Samuel. (1968). Political order in changing societies. New Haven, Yale University Press.

Jessop, B. (2001). Bringing the State Back In (Yet Again): Reviews, revisions, rejections, andre directions. International Review of Sociology. 11(2), pp.149-173.

Kaufmann, Daniel; Kraay, Art; Mastruzzi, Massimo. (2004). Governance Matters III: Governance Indicators for 1996-2002. World Bank Policy Research. Working Paper 3106. World Bank, Washington, DC.

Kettl, Donald. (2006). Public Bureaucracies. IN: R.A. Rhodes; S. Binder B. Rockman (eds.) The Oxford Handbook of Political Institutions. Oxford University Press, pp. 366-383.

Klijn, E. H.; Koppenjan, J. F. M. (2000). Public Management and Policy Networks, Public Management: An International Journal of Research and Theory, 2:2, 135-158.

Kohli, A. (2004). State-Directed Development: Political Power and Industrialization in the Global Periphery, Cambridge University Press. . (2010). State capacity for development. New York: UNDP, (Global Event Working Paper).

Levi, M. (1988). Of Rule and Revenue. Berkeley: University of California Press.

Lipsky, Michael. (1980). Street-Level Bureaucracy, Dilemmas of the Individual in Public Services. New York, Russell Sage Foundation. 
Lopez, Felix; Praça, Sérgio. (2015). Critérios e Lógicas de Nomeação para o Alto Escalão da Burocracia Federal Brasileira. IN: Cargos de confiança no presidencialismo de coalizão brasileiro / organizador: Felix Garcia Lopez. - Brasília : IPEA.

Lotta, Gabriela Spanghero. (2010). Implementação de políticas públicas: o impacto dos fatores relacionais e organizacionais sobre a atuação dos burocratas de nível de rua no Programa Saúde da Família. 2010. Tese (Doutorado em Ciência Política) - Faculdade de Filosofia, Letras e Ciências Humanas, Universidade de São Paulo, São Paulo.

; Oliveira, V. (2015). Implementando uma inovação: a burocracia de médio escalão do Programa Bolsa Família. In: Pedro Cavalcante e Gabriela Lotta. (Org.). Burocracia de Médio Escalão: perfil, trajetória e atuação. 1ed.Brasília: ENAP.

Lotta, G. S.; Pires, Roberto; Oliveira, V. E. (2014). Burocratas de médio escalão: novos olhares sobre velhos atores da produção de políticas públicas. Revista do Serviço Público, v. 64, p. 463-492.

Mann, M. (1993). The Sources of Social Power: The rise of classes and nation-states. 1760-1914. Cambridge, UK: Cambridge University Press.

Marenco, André; Strohschoen, Maria Tereza Blanco; Joner, William. (2016). Para além de sístoles e diástoles: capacidade estatal, receita e policies nos municípios brasileiros. $10^{\circ}$ Encontro da Associação Brasileira de Ciência Política.

Marin, Pedro de Lima. (2016). Sistemas de Gestão para Resultados no Setor Público: Intersecções entre política, governança e desempenho nas prefeituras de Rio de Janeiro e São Paulo. Tese (Doutorado) - Escola de Administração de Empresas de São Paulo da Fundação Getulio Vargas (FGV-EAESP). São Paulo - p.234.

Marques, Eduardo Cesar. (2003). Redes sociais, Instituições e Atores Políticos no Governo da Cidade de São Paulo. Anablume e Fapesp, São Paulo.

. (2013). As políticas públicas na Ciência Política. In: Eduardo Marques; Carlos Aurélio Pimenta de Faria. (Org.). A política pública como campo multidisciplinar. São Paulo: Editora Unesp, p. 23-46.

Meckling, Jonas; Nahm, Jonas (2018). The power of process: State capacity and climate policy. Governance. Vol. 31, pp. 1-17.

Mommsen, W. J. (1989). The political and social theory of Max Weber: collected essays. Chicago, The University of Chicago Press.

Nistotskaya, Marina; Cingolani, Luciana. (2016). Bureaucratic structure, regulatory quality, and entrepreneurship in a comparative perspective: cross-sectional and panel data evidence. Journal of Public Administration Research and Theory, Vol.26, Issue 3, pp. 519-534.

Oliveira, V. E.; Abrúcio, F. L. (2011). Entre a política e a burocracia: a importância dos burocratas de nível médio para a produção de políticas públicas em saúde e educação. Artigo apresentado no 35o. Encontro Anual da ANPOCS. Caxambu, MG.

Oliveira, Vanessa Elias; Lotta, Gabriela. (2017). De qual burocracia as políticas públicas intersetoriais e federativas precisam?: análise das competências requeridas à atuação dos gestores do programa Bolsa Família- Cadernos, 50. Brasília: Enap.

Ottervik, Mattias. (2013). Conceptualizing and Measuring State Capacity: Testing the Validity of Tax Compliance as a Measure of State Capacity. The Quality of Governance Institute Working Paper Series No. 20.

Perdicaris, Priscilla Reinisch. (2012). Contratualização de Resultados e Desempenho no Setor Público: A experiência do Contrato Programa nos hospitais da Administração Direta no Estado de São Paulo. Tese (Doutorado) Escola de Administração de Empresas de São Paulo da Fundação Getúlio Vargas (FGV-EAESP). São Paulo- SP. $133 \mathrm{p}$.

Peters, Guy. (2001). La política de la burocracia. Mexico: Fondo de Cultura Económica.

Pires, Roberto. (2009). Estilos de Implementação e Resultados de Políticas Públicas: fiscais do trabalho e o cumprimento da lei trabalhista no Brasil. Dados, v. 52, p.735-769. 
. (2012). Burocracias, gerentes e suas 'histórias de implementação': narrativas do sucesso e fracasso de programas federais. in: Faria, C. A. P. (Org.) Implementação de Políticas Públicas: teoria e prática. Belo Horizonte: Editora PUC Minas, p.182-220.

(2015). Por dentro do PAC: dos arranjos formais às interações e práticas dos seus operadores. In: Pedro Cavalcante; Gabriela Lotta. (Org.). Burocracia de médio escalão: perfil, trajetória e atuação. 1 ed. Brasília: ENAP, v. 1 , p. 177-222.

- (2016). A implementação de políticas públicas nos encontros entre burocratas e destinatários: contribuições da sociologia do guichê. $40^{\circ}$ Encontro Anual da ANPOCS. Mesa Redonda 03. Caxambu.

; Gomide, Alexandre de Ávila. (2016). Governança e capacidades estatais: uma análise comparativa de programas federais. Rev. Sociol. Polit., v. 24, n. 58, p. 121-143, jun.

(2014). Capacidades estatais e democracia: arranjos institucionais de políticas públicas. Brasília: IPEA.

Prado, Maurício Almeida. (2012). O Processo de Implementação de Reformas Gerenciais na Educação Básica: São Paulo (2007-2010) e Minas Gerais (2004-2010) em Perspectiva Comparada. Tese (Doutorado) - Fundação Getúlio Vargas na Escola de Administração de Empresas de São Paulo. São Paulo- SP. 355 p.

Pressman, Jeffrey L; Wildavsky, Aaron. (1984). Implementation (3rd edition). University of California Press. London.

Rodrigues, Luciana de Oliveira; Araujo, Jair Andrade; Guedes, João Paulo Martins; Silva, Maria Micheliana da Costa. Mensuração da desigualdade educacional entre os Municípios Nordestinos. Rev. econ. contemp., Jan 2017, vol.21, no.1.

Rotberg, Robert I. (2003). Failed States, Collapsed States, Weak States: Causes and Indicators. In: Rotberg, Robert I (Org). State Failure and State Weakness in a Time of Terror. Washington D.C.: Brookings Institution Press.

Rotberg, Robert I. (2004). The Failure and Collapse of Nation-States: Breakdown, Prevention, and Repair. In: When States Fail: Causes and Consequence. edited by Robert I. Rotberg. Princeton: Princeton University Press.

Rothstein, Bo. (2011). The Quality of Government: Corruption, Social Trust, and Inequality in International Perspective. Chicago: University of Chicago Press.

; Stolle, Dietlind. (2008). The State and Social Capital: an Institutional Theory of Generalized Trust. Comparative Politics.

Rua, Maria das Graças. (1998). Análise de Política Públicas: Conceitos Básicos. In: O Estudo da Política: Tópicos Selecionados, Brasília - DF: Paralelo 15.

Santos, Eli Izidro dos; Carvalho, Ícaro Célio Santos de; Barreto, Ricardo Candéa Sá. (2017). Pobreza multidimensional no estado da Bahia: uma análise espacial a partir dos censos de 2000 e 2010. Rev. Adm. Pública. Mar, vol.51, no. 2, p.240-263.

Silberman, Bernard. (1993). Cages of Reason: The Rise of the Rational State in France, Japan, the United States, and Great Britain. Chicago: University of Chicago Press.

Scarpin, Jorge Eduardo; Slomski, Valmor. (2007). Estudo dos fatores condicionantes do índice de desenvolvimento humano nos municípios do estado do Paraná: instrumento de controladoria para a tomada de decisões na gestão governamental. Rev. Adm. Pública. Out, vol.41, no.5, p.909-933.

Silveira Neto, Raul da Mota; Menezes, Tatiane Almeida de. (2010). Nível e evolução da desigualdade dos gastos familiares no Brasil: uma análise para as regiões metropolitanas no período 1996 a 2003. Estud. Econ., Jun. vol.40, no.2, p.341-372.

Skocpol, T. (1985). Bringing the State Back In: Strategies of analysis in current research. In: P. Evans; D. Rueschemayer e T. Skocpol (Orgs.). Bringing the State Back in. Cambridge, UK: Cambridge University Press.

(1979). States and Social Revolutions: A comparative analysis of France, Russia, and China. Cambridge, UK: Cambridge University Press.

; Finegold, K. (1982). State Capacity and Economic Intervention in the Early New Deal. Political Science Quarterly, 97(2), pp.255-278.

Souza, Celina. (2015). Capacidade burocrática no Brasil e na Argentina: Quando a política faz a diferença. Texto para discussão -Instituto de Pesquisa Econômica Aplicada -IPEA. Brasília: Rio de Janeiro: Ipea. 
(2017). Modernização do Estado e construção de capacidade burocrática para a implementação de políticas federalizadas. Rev. Adm. Pública. Rio de Janeiro, v. 51, n. 1, p. 27-45, fev.

Suzuki, Kohei; Avellaneda, Claudia. (2018). New public management and municipal performance: Do NPM reforms boost performance? QoG Working Paper Series 2018:12, December.

Thomas, Melissa A. (2009). What Do the Worldwide Governance Indicators Measure? European Journal of Development Research. 22 (1):31-54.

Tilly, Charles. (1975). The Formation of National States in Europe. Princeton: Princeton University. (2007). Democracy. Cambridge University Press.

Veiga, Laura da; Bronzo, Carla. (2014). Estratégias intersetoriais de gestão municipal de serviços de proteção social: a experiência de Belo Horizonte. Rev. Adm. Pública, Rio de Janeiro, v. 48, n. 3, p. 595-620, Jun.

Wade, R. (1990). Governing the market: economic theory and the role of government in Taiwan's industrialization. Princeton: Princeton University Press.

Walker, Richard M; Brewer, Gene; Boyne, George; Avellaneda, Claudia N. (2011). Market orientation and public service performance: new public management gone mad? Public Administration Review. 71 (5):707-717.

Wang, Shaoguang. (2003). The Problem of State Weakness. Journal of Democracy. 14(1): 36-42.

Wilson, James Q. (1968). Varieties of Police Behavior: The Management of Law and Orderin Eight Communities. Cambridge, Harvard University Press.

(1989). Bureaucracy. What government agencies do and why they do it. Basic Books.

Zittoun, P. (2014). The political Process of Policymaking, A pragmatic approach on Public Policy. Palgrave-McMilan.

\section{Anexo A: Nomes dos municípios analisados IDH-M.}

Rio Fortuna, Treze Tílias, Iomerê, Holambra, Três Arroios, Ipiranga do Sul, Quatro Pontes, Saltinho, Santa Cruz da Conceição, Cândido Rodrigues, Lagoa dos Três Cantos, Luzerna, Fernando de Noronha, Nova Araçá, Casca, Salto Veloso, Horizontina, Pinhalzinho, Águas da Prata, Bombinhas, Lacerdópolis, Corupá, Vista Alegre do Prata, Cocal do Sul, Braço do Trombudo, Seara, Piratininga, Nova Bréscia, Selbach, Victor Graeff, Pinheiro Preto, Itaú de Minas, Itapiranga, Trombudo Central, Pedrinhas Paulista, Ouro, Cruzália, Siderópolis, Treviso, Massaranduba, Paraí, Auriflama, Santana da Ponte Pensa, Sebastianópolis do Sul, Serra Alta, Aratiba, Monções, Sales Oliveira, Santa Salete, Itá, Lajeado Grande, Maracaí, Macatuba, Presidente Castello Branco, Oriente, Armazém, Ouroeste, Tupi Paulista, São Carlos, Maracajá, Regente Feijó, Bilac, Nova Boa Vista, Nova Veneza, Novo Xingu, Orindiúva, Capivari do Sul, Peritiba, São Simão, Ivatuba, Cedral, Alumínio, Poloni, São Domingos, Não-Me-Toque, Espumoso, Colinas, Nova Erechim, Nova Europa, Rosana, Santo Antônio do Palma, Rondinha, Cerro Largo, Arroio Trinta, São Valentim do Sul, Montauri, São Pedro do Butiá, São Domingos do Sul, Buritama, Barra Funda, Gabriel Monteiro, Fagundes Varela, Serranópolis do Iguaçu, David Canabarro, Saldanha Marinho, Boa Vista do Buricá, Entre Rios do Oeste, Itapoá, Indiana, Taió, Curral Novo do Piauí, Alvarães, Milagres do Maranhão, Mirante, Flexeiras, Japorã, Poço das Trincheiras, Carneiros, Pavussu, Santa Filomena do Maranhão, Olho dÁgua do Casado, Massapê do Piauí, Lagoa do Ouro, Poço Dantas, Santa Cecília, Campo Grande, Nova Itarana, Macajuba, Jaicós, Cuité de Mamanguape, Cajari, São João do Arraial, Cacimbas, Juruá, Joaquim Pires, Japurá, Novo Lino, Governador Newton Bello, Damião, Ribeira do Piauí, Amapá do Maranhão, Serrano do Maranhão, Santana do Mundaú, Aroeiras do Itaim, Brejo de Areia, Santo Amaro do Maranhão, Itaipava do Grajaú, Senador Rui Palmeira, Ibateguara, Lamarão, Santa Rosa do Purus, Belo Monte, São João do Soter, Jutaí, São Raimundo do Doca Bezerra, São Roberto, São João da Fronteira, Queimada Nova, Brejo do Piauí, Umburanas, Pau DArco do Piauí, Casserengue, Senador José Porfírio, Gado Bravo, Pedro Alexandre, Branquinha, Belágua, Primeira Cruz, Ribeira do Amparo, Conceição do Lago-Açu, Ibiquera, Santana 
do Maranhão, Envira, São João do Carú, Paquetá, Jurema, Guaribas, Milton Brandão, Beruri, Canapi, Jacareacanga, Caraúbas do Piauí, Roteiro, Joca Marques, Olho dÁgua Grande, Vera Mendes, Tapauá, Lagoa do Barro do Piauí, Lagoa Grande do Maranhão, Marechal Thaumaturgo, Tamboril do Piauí, Recursolândia, Água Doce do Maranhão, Assunção do Piauí, Maraã, Cocal dos Alves, Pauini, Satubinha, Olivença, Jenipapo dos Vieiras, Betânia do Piauí, Caxingó, São Francisco de Assis do Piauí, Amajari, Inhapi, Itamarati, Jordão, Uiramutã, Marajá do Sena, Atalaia do Norte e Fernando Falcão.

\section{Nomes dos municípios analisados Gini.}

Itamarati, Isaías Coelho, Jequitibá, Alto Parnaíba, Santa Rosa do Purus, Uiramutã, Buritinópolis, Amajari, Pacaraima, Lizarda, Guajará, Alto Alegre, Jacuizinho, Bonfim, Pauini, Abreulândia, Mucajaí, Jordão, Normandia, São Domingos do Azeitão, Campinápolis, Jutaí, Jacareacanga, Maraã, Capivari do Sul, Água Santa, Herval, Aurora do Tocantins, Manga, Augusto Severo, Gilbués, Santa Cruz da Conceição, Itapiranga, Calçoene, Gaúcha do Norte, Brasil Novo, Campo Novo de Rondônia, São Gonçalo do Gurguéia, Campos Lindos, Jenipapo dos Vieiras, Inhapi, Mairipotaba, Jussara, Miraguaí, Jardim Alegre, Amapá, Cantá, Japorã, Alto Paraíso de Goiás, Pracuúba, Aguiar, Paranã, Tartarugalzinho, Primavera, Campo Alegre do Fidalgo, Belo Monte, Beruri, Atalaia do Norte, Juruaia, Nova Santa Helena, Divinópolis do Tocantins, Jaú do Tocantins, Nova Brasilândia, Taguatinga, Redentora, São João do Pacuí, Miraíma, Paranhos, São Félix do Tocantins, Amaturá, Cumaru do Norte, Poço das Trincheiras, Envira, Recursolândia, Itaipulândia, Pouso Alto, Rio Azul, Palmácia, Ipaumirim, Ponte Alta do Bom Jesus, Seringueiras, Flores de Goiás, Itanagra, Centenário, Rodrigues Alves, Formosa da Serra Negra, Cajueiro da Praia, Cajari, Belágua, Treze Tílias, Três Arroios, Fortaleza dos Valos, Campos de Júlio, Novo Barreiro, Minas do Leão, Peixe, São José do Xingu, Nova Bandeirantes, Lucrécia, Monte do Carmo, Salvador das Missões, Harmonia, Linha Nova, Laurentino, São José do Inhacorá, Mato Leitão, Itambé, Bady Bassitt, Mendonça, Dolcinópolis, Alfredo Marcondes, Santa Clara do Sul, Benedito Novo, Morro da Fumaça, Floresta, Nova Independência, Valentim Gentil, Embaúba, Ipiguá, Treze de Maio, Coronel Pilar, Agrolândia, São João das Duas Pontes, Narandiba, Arvoredo, Irapuã, Ubiretama, Brochier, Nova América da Colina, Araújos, Areiópolis, Marilena, Timbaúba dos Batistas, Santa Salete, Montauri, Fagundes Varela, Vanini, Turiúba, União Paulista, Nova Trento, Vista Alegre do Alto, Nova Luzitânia, Imigrante, Ascurra, Santópolis do Aguapeí, Cachoeira Dourada, Vitória Brasil, Indianópolis, Vespasiano Corrêa, Jaci, Gavião Peixoto, Nantes, Japurá, Perdigão, Luiziânia, Travesseiro, Alto Alegre, Ubarana, Santo Antônio do Caiuá, Araricá, Albertina, Treviso, Massaranduba, Dois Lajeados, Anta Gorda, Onda Verde, São João do Itaperiú, Tamboara, Águas Mornas, Palmares Paulista, Nova Aliança do Ivaí, Doutor Pedrinho, Nova Canaã Paulista, Florestópolis, Maratá, Bandeira do Sul, Paverama, Iomerê, Saudades, Monte Belo do Sul, Westfália, São João do Pau dAlho, Elisiário, Pedras Grandes, Pitangueiras, Nossa Senhora das Graças, Nova Pádua, Picada Café, Santa Rosa de Lima, Presidente Lucena, Morro Reuter, Vila Flores, Córrego Fundo, Tupandi, Campestre da Serra, Santa Maria do Herval, São Vendelino, Vale Real, Alto Feliz, Botuverá e São José do Hortêncio.

\section{Notas}

[1] Categorização realizada por Gabriela Lotta, durante palestra proferida no Seminário Internacional Construindo Capacidades Estatais: os Desafios do Serviço Público, organizado pela Enap (Escola Nacional de Administração Pública) em 2017.

[2] Sobre uso dos indicadores IDH-M e Gini, ver Scarpin \& Slomski (2007), Silveira Neto \& Menezes (2010), Rodrigues et al. (2017), Santos et al. (2017).

[3] As informações sobre distribuição de servidores públicos municipais segundo vínculo e escolaridade não estão disponíveis para todos os anos na Base MUNIC/IBGE. Desta forma, a escolha dos anos levou em conta sua disponibilidade. 
André Luis Rabelo Cardoso, et al. Qualidade Burocrática e Performance Estatal: Desvendando a caixa...

CC BY-NC-ND 